\title{
Stress causes and outcomes statistical analysis
}

\begin{abstract}
In medical term "stress" is the change in mental and physical status of the body caused by factors including physical, mental or emotional tension. Stress can cause a lot of clinical and psychological conditions such as depression and anxiety. Stress complications are common in people experiencing very high levels of negative stress leading to weakness and overwhelming. It could be a result of continuous stress incidence due to different scenarios. "Stressed out" is a phenomenon that is highly increasing and affecting almost everyone. Stress is all over around us in every situation and every day, but the importance is how to handle the changes and not experiencing the changes as good or bad. Generally, changes in our life as we go on happen all the time and our reaction to these changes is what causes stress. Life changes such as studying a new level of education (e.g Masters), getting new job, losing a job, moving to new romantic partner, or family problems. These changes are new stressful events considered either positive and life-enhancing or negative. The degree of stress varies between high and low, people don't like to experience the extremes of stress. However; the capacity of each person to overcome the changes in feeling varies. On the other hand, some people enjoy being overwhelmed in important challenging changes and some don't like the total absence of stress. In conclusion, people tend to reach the middle ground a balance between a lack of stress and too much stress, moreover; human by nature want to live in a calm atmosphere without experiencing high levels of stress. In this paper, we did an empirical study by collected data from health sciences post grad students who work and study using a survey that was administered by smart phone applications. The main idea is to detect the causes that lead to stress and could worsen the mental and physical activity of the body.
\end{abstract}

Volume 7 Issue 4 - 2018

Seifedine Kadry,' Maryam Kbaysi, ${ }^{2}$ Suhad AlSafadi, ${ }^{2}$ Dareen Al-Bakri

'Department of mathematics and statistics, Faculty of Science, Beirut Arab University, Lebanon

${ }^{2}$ Faculty of Health Sciences, Beirut Arab University, Lebanon

Correspondence: Seifedine Kadry, Department of mathematics and computer science, Faculty of Science, Beirut Arab University, Beirut, Lebanon, Tel +96/3 7005 I2, Email skadry@gmail.com

Received: May 19, 2018 | Published: August 15, 2018

\section{Literature review}

Everyone has different stress triggers, work stress tops the list. According to surveys $40 \%$ of United States workers admit to experience office stress, and one -quarter say work is the biggest source of stress in their lives. ${ }^{1}$ Stress in everyday terms is a feeling that people have when they are overloaded and struggling to cope with demands which are related to finances, work, relationships and other situations. ${ }^{2}$ According to the annual stress survey conducted by the American Psychological Association (APA) average stress levels in the United States (U.S) raised from 4.9 to 5.1 on a scale from 1 to 10 in 2015 the main reasons given are employment and money. ${ }^{3}$

Another study was done in accordance with international coordination of labor conditions a Japanese campaign advocating less work finally got under way recently in the form of work-reducing policies of the government to prevent occupational and stress-related diseases. ${ }^{4}$ However, long work hours among intermediate managers, who are key persons in most organizations in Japanese industry, are still considered to be prevalent. ${ }^{5,6}$ This study was conducted to examine the work hours of intermediate managers and clarify the effects of long work hours on the life-style, subjective stress, and subjective quality of life among them.

Questionnaires were administered concerning, life-styles, subjective stress, and subjective quality of life to 3870 heads of a division or a section and 2666 foremen in 110 firms in Japan. The prevalence of $>$ or $=10$ work hours was $69.7 \%$ for the divisional or sectional heads and $53.2 \%$ for the foremen. Long work hours had significant effects on the managers' life-style, such as sleeping pattern and regularity of daily life and meals. The divisional or sectional managers with long work hours perceived higher stress [odds ratio (OR) 2.51, 95\% confidence interval (95\% CI) 2.17-2.90] and lower quality of life (OR 1.17, 95\% CI 1.02-1.36) than those who worked relatively short hours. The foremen with long work hours perceived higher stress (OR 2.35, 95\% CI 2.01-2.75) and lower quality of life (OR 1.26, 95\% CI 1.08-1.46) than those who worked relatively short hours. In conclusion, long work hours may be associated with poorer life-style, higher stress, and lower quality of life among managers at the intermediate level.

\section{Data collection}

The data was collected from health sciences department post grad students who work and study using a survey that was administered by smart phone applications. The main idea of this survey is to detect the causes that lead to stress and could worsen the mental and physical activity of the body.

a. Sample size, $n=100$ students who study and work mainly filled the survey.

b. Data was entered, tabulated and analyzed using SPSS Software.

c. The questionnaire design consists of one types of questions:

d. Close ended questions in which the respondents are given list of predetermined responses from which to choose their answer.

e. The variables are designed to answer the research questions.

The variables in the data set are:

1. Stress: yes or no

2. Lack time to exercise: yes or no

3. Lack time to socialize: yes or no

4. Overworking: yes or no 
5. Unmotivated: yes or no

6. Overeating: yes or no

7. Loss of self-control: yes or no

8. Comforts you when you are stressed smoking or Eating or Listening to music or Exercise or Going out with friends

9. Solution for work overload related stress: leave the work or Manage your time or Decrease working hours or Go for a holiday

\section{Descriptive statistics}

Stress: $56 \%$ of the subjects were stressed while $44 \%$ were not stressed (Table 1) (Figure 1).

Table I Stress

\begin{tabular}{lllll}
\hline & Frequency & Percent & $\begin{array}{l}\text { Valid } \\
\text { percent }\end{array}$ & $\begin{array}{l}\text { Cumulative } \\
\text { percent }\end{array}$ \\
\hline Yes & 56 & 56 & 0 & 56 \\
No & 44 & 44 & 44 & 100 \\
Total & 100 & 100 & 100 & \\
\hline
\end{tabular}

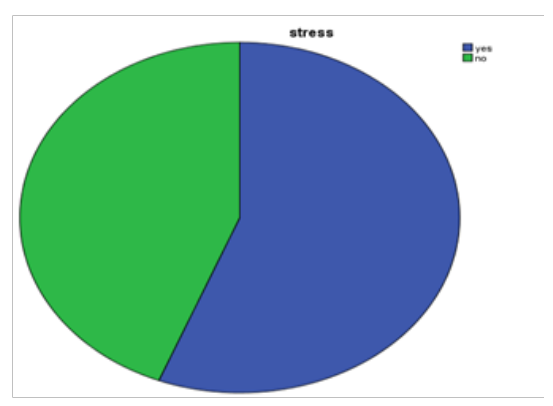

Figure I Stress.

Lack time to exercise: $61 \%$ of the subjects lack time to exercise while 39\% have time to exercise (Table 2) (Figure 2).

Table 2 Exercise time

\begin{tabular}{llllll}
\hline & & Frequency & Percent & $\begin{array}{l}\text { Valid } \\
\text { percent }\end{array}$ & $\begin{array}{l}\text { Cumulative } \\
\text { percent }\end{array}$ \\
\hline Valid & Yes & 61 & 61 & 61 & 61 \\
& No & 39 & 39 & 39 & 100 \\
& Total & 100 & 100 & 100 & \\
\hline
\end{tabular}

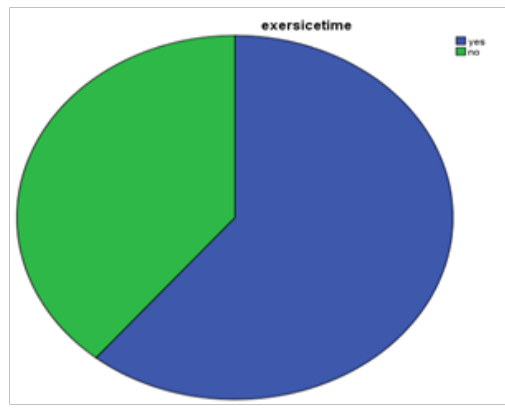

Figure 2 Exercise time.
Socializing time: $55 \%$ of the subjects lack time to socialize while $45 \%$ have time to socialize (Table 3 ) (Figure 3 ).

\section{Table 3 Socialize}

\begin{tabular}{|c|c|c|c|c|c|}
\hline & & Frequency & Percent & $\begin{array}{l}\text { Valid } \\
\text { percent }\end{array}$ & $\begin{array}{l}\text { Cumulative } \\
\text { percent }\end{array}$ \\
\hline \multirow[t]{3}{*}{ Valid } & Yes & 55 & 55 & 55 & 55 \\
\hline & No & 45 & 45 & 45 & 100 \\
\hline & Total & 100 & 100 & 100 & \\
\hline & & & sociall & & \\
\hline
\end{tabular}

Figure 3 Socialize.

Sleepind disturbance: $55 \%$ of the subjects suffer from sleeping disturbance while $45 \%$ sleep normally (Table 4) (Figure 4).

Table 4 Sleep

\begin{tabular}{llllll}
\hline & & Frequency & Percent & $\begin{array}{l}\text { Valid } \\
\text { percent }\end{array}$ & $\begin{array}{l}\text { Cumulative } \\
\text { percent }\end{array}$ \\
\hline Valid & Yes & 55 & 55 & 55 & 55 \\
& No & 45 & 45 & 45 & 100 \\
& Total & 100 & 100 & 100 & \\
\hline
\end{tabular}

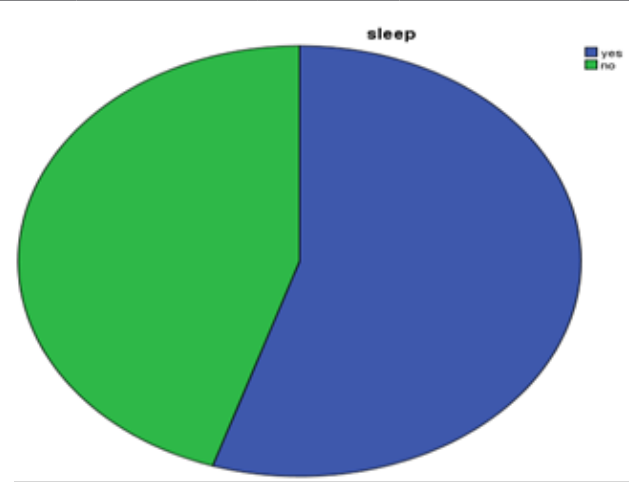

Figure 4 Sleep.

Unmotivated: $53 \%$ of the subjects are unmotivated while $47 \%$ feel normal (Table 5) (Figure 5).

Table 5 Unmotivated

\begin{tabular}{llllll}
\hline & & Frequency & Percent & $\begin{array}{l}\text { Valid } \\
\text { percent }\end{array}$ & $\begin{array}{l}\text { Cumulative } \\
\text { percent }\end{array}$ \\
\hline Valid & Yes & 53 & 53 & 53 & 53 \\
& No & 47 & 47 & 47 & 100 \\
& Total & 100 & 100 & 100 & \\
\hline
\end{tabular}




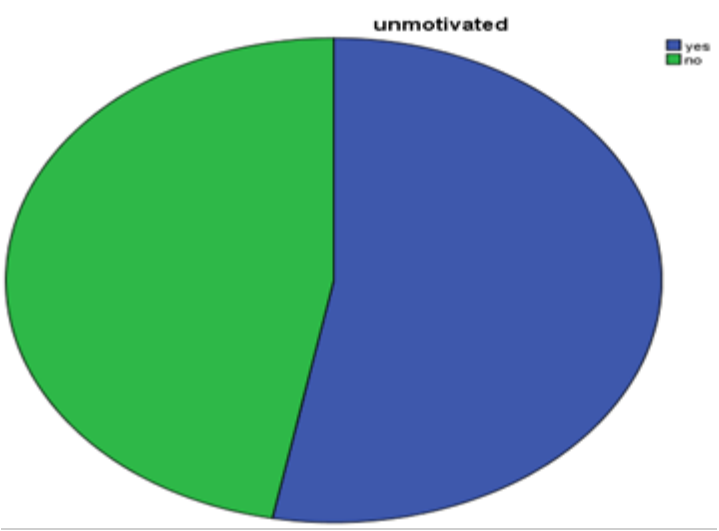

Figure 5 Unmotivated.

Work overload: $71 \%$ of the subjects suffer from work overload while $29 \%$ do not suffer (Table 6) (Figure 6).

Table 6 Work overload

\begin{tabular}{llllll}
\hline & & Frequency & Percent & $\begin{array}{l}\text { Valid } \\
\text { percent }\end{array}$ & $\begin{array}{l}\text { Cumulative } \\
\text { percent }\end{array}$ \\
\hline Valid & Yes & 71 & 71 & 71 & 71 \\
& No & 29 & 29 & 29 & 100 \\
& Total & 100 & 100 & 100 & \\
\hline
\end{tabular}

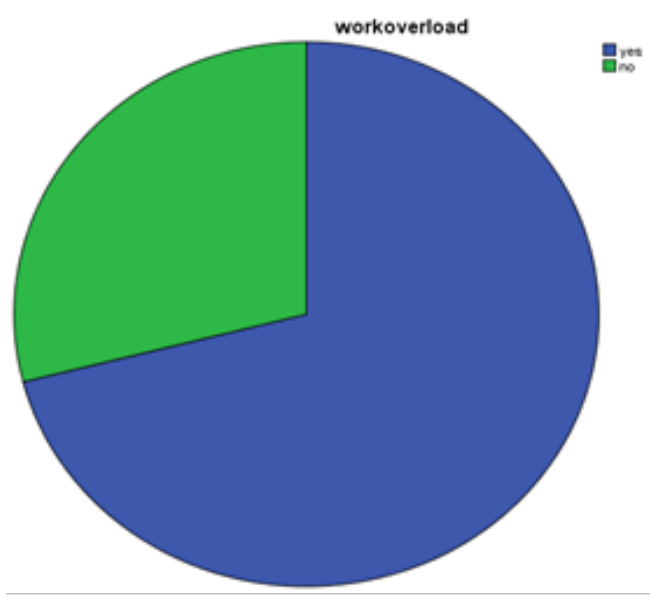

Figure 6 Work overload.

Overeat: $53 \%$ suffer from overeating while $73 \%$ have normal eating habits (Table 7) (Figure 7).

Table 7 Overeating

\begin{tabular}{llllll}
\hline & & Frequency & Percent & $\begin{array}{l}\text { Valid } \\
\text { percent }\end{array}$ & $\begin{array}{l}\text { Cumulative } \\
\text { percent }\end{array}$ \\
\hline Valid & Yes & 53 & 53 & 53 & 53 \\
& No & 47 & 47 & 47 & 100 \\
& Total & 100 & 100 & 100 &
\end{tabular}

Self-control: $40 \%$ of the subjects have loss of self-control while $60 \%$ are normal (Table 8) (Figure 8).

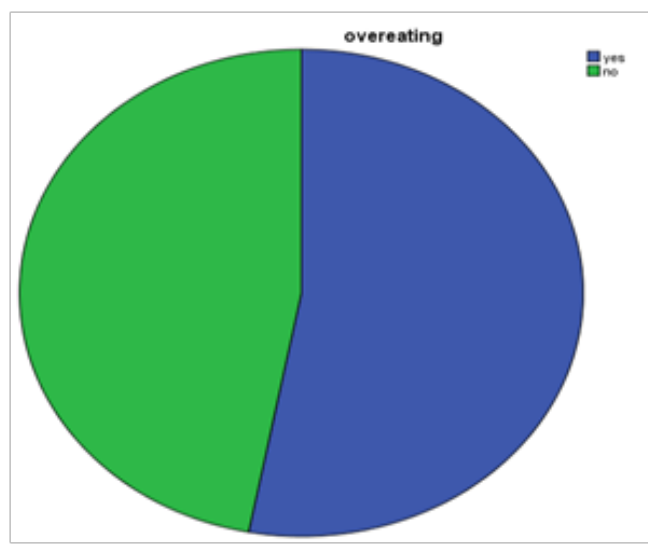

Figure 7 Overeating.

Table 8 Self-control

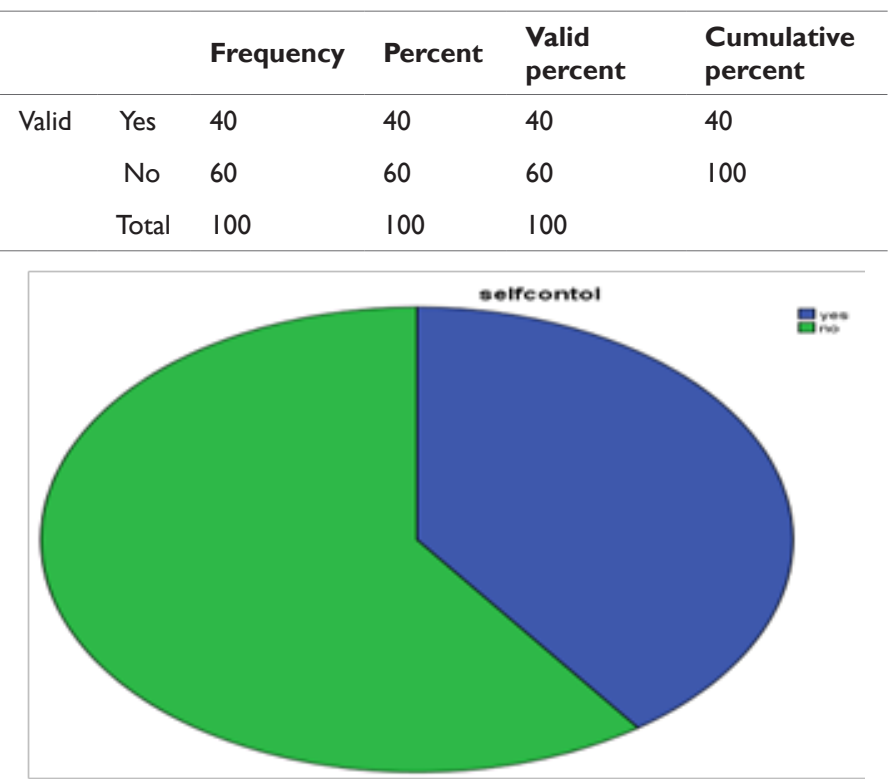

Figure 8 Self-control.

Comfort when stressed: Here we can assume that listening to music is the most used way to reduce stress (Table 9) (Figure 9).

Table 9 Comfort

\begin{tabular}{|c|c|c|c|c|c|}
\hline & & Frequency & Percent & $\begin{array}{l}\text { Valid } \\
\text { percent }\end{array}$ & $\begin{array}{l}\text { Cumulative } \\
\text { percent }\end{array}$ \\
\hline \multirow[t]{6}{*}{ Valid } & Smoking & 15 & 15 & 15 & 15 \\
\hline & Eating & 16 & 16 & 16 & 31 \\
\hline & $\begin{array}{l}\text { Listening } \\
\text { to music }\end{array}$ & 36 & 36 & 36 & 67 \\
\hline & Exercise & 18 & 18 & 18 & 85 \\
\hline & $\begin{array}{l}\text { Going } \\
\text { out with } \\
\text { friends }\end{array}$ & 15 & 15 & 15 & 100 \\
\hline & Total & 100 & 100 & 100 & \\
\hline
\end{tabular}




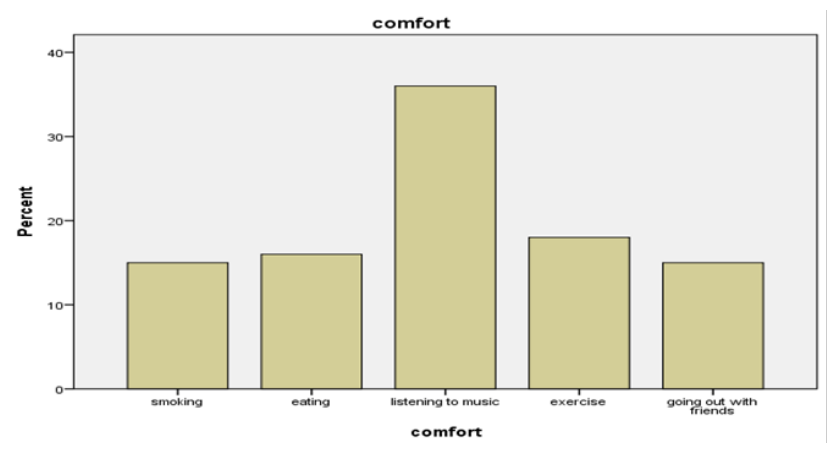

Figure 9 Comfort.

Management of stress due to work overload: Here we can see that people preferred lowering working hour as an effective way to reduce work related stress (Table 10) (Figure 10).

\section{Table 10 Manage}

\begin{tabular}{lllll}
\hline & Frequency & Percent & $\begin{array}{l}\text { Valid } \\
\text { percent }\end{array}$ & $\begin{array}{l}\text { Cumulative } \\
\text { percent }\end{array}$ \\
\hline Leave your work & 19 & 19 & 19 & 19 \\
$\begin{array}{l}\text { Mange your time } \\
\text { Lower work }\end{array}$ & 20 & 20 & 20 & 39 \\
$\begin{array}{l}\text { hours } \\
\text { Go for a holiday }\end{array}$ & 43 & 43 & 43 & 82 \\
Total & 18 & 18 & 18 & 100 \\
\hline
\end{tabular}

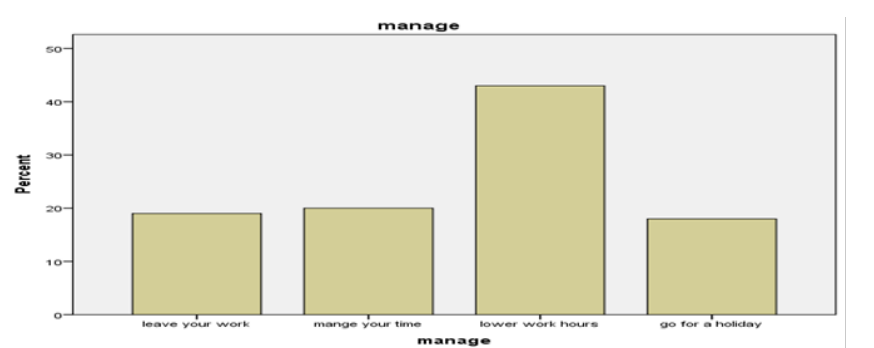

Figure 10 Manage.

\section{Inferential statistics}

\section{Research questions}

\section{Does lacking time to exercise cause stress?}

$\mathrm{H} 0=$ lacking time to exercise does not cause stress.

$\mathrm{Ha}=$ lacking time to exercise causes stress (Table 11).

According to the chi square test the $\mathrm{p}$ value $=0.00$ and when the $\mathrm{p}$ value is lower than 0.05 it's considered significant. Therefore we accept the Ha and we reject the H0. So we can conclude that lacking time to exercise causes stress.

\section{Does sleep disturbance cause stress?}

$\mathrm{H} 0=$ sleep disturbance does not cause stress.

$\mathrm{Ha}=$ sleep disturbance causes stress (Table 12).

According to the chi square test the $\mathrm{p}$ value $=0.044$ and when the $\mathrm{p}$ value is lower than 0.05 it's considered significant. Therefore we accept the Ha and we reject the H0. So we can conclude that sleeping disturbance causes stress.

Table I I Stress* exercise time Cross tabulation

\begin{tabular}{lcccc}
\hline Count & & \multicolumn{3}{c}{} \\
& & \multicolumn{2}{c}{ Exercise time } & Total \\
& & Yes & No & \\
\hline Stress & Yes & 44 & 12 & 56 \\
& No & 17 & 27 & 44 \\
Total & & 61 & 39 & 100 \\
\hline
\end{tabular}

\begin{tabular}{|c|c|c|c|c|c|}
\hline \multicolumn{6}{|l|}{ Chi-Square tests } \\
\hline & Value & df & $\begin{array}{l}\text { Asymp. } \\
\text { Sig. } \\
\text { (2-sided) }\end{array}$ & $\begin{array}{l}\text { Exact } \\
\text { Sig. } \\
\text { (2-sided) }\end{array}$ & $\begin{array}{l}\text { Exact } \\
\text { Sig. } \\
\text { (I-sided) }\end{array}$ \\
\hline $\begin{array}{l}\text { Pearson Chi- } \\
\text { Square }\end{array}$ & $16.518^{\mathrm{a}}$ & 1 & 0 & & \\
\hline $\begin{array}{l}\text { Continuity } \\
\text { Correction }^{\mathrm{b}}\end{array}$ & 14.882 & 1 & 0 & & \\
\hline $\begin{array}{l}\text { Likelihood } \\
\text { Ratio }\end{array}$ & 16.852 & 1 & 0 & & \\
\hline Fisher's Exact Test & & & & 0 & 0 \\
\hline $\begin{array}{l}\text { Linear-by-Linear } \\
\text { Association }\end{array}$ & 16.353 & 1 & 0 & & \\
\hline $\begin{array}{l}N \text { of Valid } \\
\text { Cases }\end{array}$ & 100 & & & & \\
\hline
\end{tabular}

Table 12 Stress* Sleep Cross tabulation

\begin{tabular}{|c|c|c|c|c|}
\hline \multicolumn{5}{|c|}{ Count } \\
\hline & & \multicolumn{2}{|c|}{ Sleep } & \multirow{2}{*}{ Tota } \\
\hline & & Yes & No & \\
\hline \multirow[t]{2}{*}{ Stress } & Yes & 36 & 20 & 56 \\
\hline & No & 19 & 25 & 44 \\
\hline Total & & 55 & 45 & 100 \\
\hline
\end{tabular}

\section{Chi-Square tests}

\begin{tabular}{|c|c|c|c|c|c|}
\hline & Value & df & $\begin{array}{l}\text { Asymp. } \\
\text { Sig. } \\
\text { (2-sided) }\end{array}$ & $\begin{array}{l}\text { Exact } \\
\text { Sig. } \\
\text { (2-sided) }\end{array}$ & $\begin{array}{l}\text { Exact } \\
\text { Sig. } \\
\text { (I-sided) }\end{array}$ \\
\hline $\begin{array}{l}\text { Pearson Chi- } \\
\text { Square }\end{array}$ & $4.434^{\mathrm{a}}$ & I & 0.035 & & \\
\hline $\begin{array}{l}\text { Continuity } \\
\text { Correction }^{\mathrm{b}}\end{array}$ & 3.622 & 1 & 0.057 & & \\
\hline Likelihood Ratio & 4.455 & I & 0.035 & & \\
\hline Fisher's Exact Test & & & & 0.044 & 0.028 \\
\hline $\begin{array}{l}\text { Linear-by-Linear } \\
\text { Association }\end{array}$ & 4.39 & I & 0.036 & & \\
\hline $\mathrm{N}$ of Valid Cases & 100 & & & & \\
\hline
\end{tabular}

a. 0 cells $(0.0 \%)$ have expected count less than 5 . The minimum expected count is 19.80 .

b. b. Computed only for a $2 \times 2$ table. 


\section{Does lacking time to socialize causes stress?}

$\mathrm{H} 0=$ lacking time to socialize does not cause stress.

$\mathrm{Ha}=$ lacking time to socialize causes stress (Table 13)

Table 13 Stress* socialize Cross tabulation

\begin{tabular}{lcccc}
\hline Count & \multicolumn{3}{c}{ Socialize } & Total \\
& & Yes & No & \\
\hline Stress & Yes & 41 & 15 & 56 \\
& No & 14 & 30 & 44 \\
Total & & 55 & 45 & 100 \\
\hline
\end{tabular}

\section{Chi-Square tests}

$\begin{array}{llllll} & \text { Value } & \text { df } & \begin{array}{l}\text { Asymp. } \\ \text { Sig. } \\ \text { (2-sided) }\end{array} & \begin{array}{l}\text { Exact Sig. } \\ \text { (2-sided) }\end{array} & \begin{array}{l}\text { Exact } \\ \text { Sig. } \\ \text { ( I-sided) }\end{array} \\ \begin{array}{l}\text { Pearson Chi- } \\ \text { Square }\end{array} & 17.060^{\mathrm{a}} & \mathrm{I} & 0 & & \\ \begin{array}{l}\text { Continuity } \\ \text { Correction }\end{array} & 15.429 & \text { I } & 0 & & \\ \begin{array}{l}\text { Likelihood Ratio } \\ \text { Fisher's Exact Test }\end{array} & 17.5 & \text { I } & 0 & & \\ \begin{array}{l}\text { Linear-by-Linear } \\ \text { Association }\end{array} & 16.89 & \text { I } & 0 & & \\ \text { N of Valid Cases } & 100 & & & & \end{array}$

a. 0 cells $(0.0 \%)$ have expected count less than 5 . The minimum expected count is 19.80

b. b. Computed only for a $2 \times 2$ table.

According to the chi square test the $\mathrm{p}$ value $=0.00$ and when the $\mathrm{p}$ value is lower than 0.05 it's considered significant. Therefore we accept the $\mathrm{Ha}$ and we reject the $\mathrm{H} 0$.

So we can conclude that lacking time to socialize causes stress.

\section{Is being unmotivated causes stress?}

$\mathrm{H} 0=$ un-motivation does not cause stress.

$\mathrm{Ha}=$ un-motivation causes stress (Table 14).

Table I 4 Stress* unmotivated

\begin{tabular}{lllll}
\hline Count & & & & \\
& & \multicolumn{2}{l}{ Unmotivated } & Total \\
& & Yes & No & \\
\hline Stress & Yes & 34 & 22 & 56 \\
& No & 19 & 25 & 44 \\
Total & & 53 & 47 & 100 \\
\hline
\end{tabular}

\begin{tabular}{|c|c|c|c|c|c|}
\hline \multicolumn{6}{|l|}{ Chi-Square tests } \\
\hline & Value & df & $\begin{array}{l}\text { Asymp. } \\
\text { Sig. } \\
\text { (2-sided) }\end{array}$ & $\begin{array}{l}\text { Exact } \\
\text { Sig. } \\
\text { (2-sided) }\end{array}$ & $\begin{array}{l}\text { Exact } \\
\text { Sig. } \\
\text { (I-sided) }\end{array}$ \\
\hline $\begin{array}{l}\text { Pearson Chi- } \\
\text { Square }\end{array}$ & $3.04 I^{\mathrm{a}}$ & 1 & 0.081 & & \\
\hline $\begin{array}{l}\text { Continuity } \\
\text { Correction }^{\mathrm{b}}\end{array}$ & 2.377 & 1 & 0.123 & & \\
\hline Likelihood Ratio & 3.052 & 1 & 0.081 & & \\
\hline Fisher's Exact Test & & & & 0.107 & 0.061 \\
\hline $\begin{array}{l}\text { Linear-by-Linear } \\
\text { Association }\end{array}$ & 3.01 & 1 & 0.083 & & \\
\hline $\mathrm{N}$ of Valid Cases & 100 & & & & \\
\hline
\end{tabular}

a. 0 cells $(0.0 \%)$ have expected count less than 5 . The minimum expected count is 20.68 .

b. b. Computed only for a $2 \times 2$ table.

According to the chi square test the $\mathrm{p}$ value $=0.107$ and when the $\mathrm{p}$ value is higher than 0.05 it's considered not significant. Therefore we accept the $\mathrm{H} 0$ and we reject the Ha. So we can conclude that being unmotivated does not cause stress.

\section{Does work overload causes stress?}

$\mathrm{H} 0=$ work overload does not cause stress.

$\mathrm{Ha}=$ work overload causes stress (Table 15).

Table 15 Stress* work overload Cross tabulation

\begin{tabular}{lllll}
\hline Count & & \multicolumn{2}{l}{} \\
& & \multicolumn{2}{c}{ Work overload } & Total \\
& & Yes & No & \\
\hline Stress & Yes & 39 & 17 & 56 \\
& No & 32 & 12 & 44 \\
Total & & 71 & 29 & 100
\end{tabular}

\section{Chi-Square tests}

\begin{tabular}{|c|c|c|c|c|c|}
\hline & Value & df & $\begin{array}{l}\text { Asymp. } \\
\text { Sig. } \\
\text { (2-sided) }\end{array}$ & $\begin{array}{l}\text { Exact } \\
\text { Sig. } \\
\text { (2-sided) }\end{array}$ & $\begin{array}{l}\text { Exact } \\
\text { Sig. } \\
\text { ( I-sided) }\end{array}$ \\
\hline $\begin{array}{l}\text { Pearson Chi- } \\
\text { Square }\end{array}$ & $.114^{a}$ & 1 & 0.736 & & \\
\hline $\begin{array}{l}\text { Continuity } \\
\text { Correction }\end{array}$ & 0.013 & I & 0.908 & & \\
\hline $\begin{array}{l}\text { Likelihood } \\
\text { Ratio }\end{array}$ & 0.114 & 1 & 0.735 & & \\
\hline
\end{tabular}

$\begin{array}{lll}\text { Fisher's Exact Test } & 0.826 & 0.456\end{array}$

Linear-

by-Linear $\quad 0.113 \quad$ I $\quad 0.737$

Association

$\mathrm{N}$ of Valid

Cases

a. 0 cells $(0.0 \%)$ have expected count less than 5 . The minimum expected count is 12.76 .

b. b. Computed only for a $2 \times 2$ table. 
According to the chi square test the $p$ value $=0.826$ and when the $\mathrm{p}$ value is higher than 0.05 it's considered not significant. Therefore we accept the $\mathrm{H} 0$ and we reject the Ha. So we can conclude that work overload does not cause stress.

\section{Does stress result in overeating?}

$\mathrm{H} 0=$ stress does not cause overeating.

$\mathrm{Ha}=$ stress causes overeating (Table 16).

Table 16 Stress* overeating Cross tabulation

\begin{tabular}{lllll}
\hline Count & & \multicolumn{2}{c}{} \\
& & \multicolumn{2}{c}{ Overeating } & Total \\
& & Yes & No & \\
\hline Stress & Yes & 32 & 24 & 56 \\
& No & 21 & 23 & 44 \\
Total & & 53 & 47 & 100 \\
\hline
\end{tabular}

Chi-Square tests

\begin{tabular}{lllll} 
Value df & $\begin{array}{l}\text { Asymp. } \\
\text { Sig. } \\
\text { (2-sided) }\end{array}$ & $\begin{array}{l}\text { Exact } \\
\text { Sig. } \\
\text { (2-sided) }\end{array}$ & $\begin{array}{l}\text { Exact } \\
\text { Sig. } \\
\text { (I-sided) }\end{array}$ \\
\hline
\end{tabular}

$\begin{array}{llll}\begin{array}{l}\text { Pearson Chi- } \\ \text { Square }\end{array} & .877^{\mathrm{a}} & \text { । } & 0.349 \\ \begin{array}{l}\text { Continuity } \\ \text { Correction }\end{array} & 0.54 & \text { । } & 0.463 \\ & & & \\ \text { Likelihood Ratio }^{\mathrm{b}} & 0.878 & \text { । } & 0.349\end{array}$

Fisher's Exact Test

0.421

0.231

Linear-by-Linear

Association

$0.868 \quad 1$

$\mathrm{N}$ of Valid Cases $\quad 100$

a. 0 cells $(0.0 \%)$ have expected count less than 5 . The minimum expected count is 20.68 .

b. Computed only for a $2 \times 2$ table

According to the chi square test the $p$ value $=0.421$ and when the $\mathrm{p}$ value is higher than 0.05 it's considered not significant. Therefore we accept the $\mathrm{H} 0$ and we reject the $\mathrm{Ha}$. So we can conclude that stress does not cause overeating.

\section{Does stress cause loss of self-control?}

$\mathrm{H} 0=$ stress does not cause loss of self-control.

$\mathrm{Ha}=$ stress causes loss of self-control (Table 17).

Table 17 Stress* self control Cross tabulation

\begin{tabular}{lllll}
\hline Count & & \multicolumn{2}{c}{} \\
& & \multicolumn{2}{c}{ Self control } & Total \\
& & Yes & No & \\
\hline Stress & Yes & 24 & 32 & 56 \\
& No & 16 & 28 & 44 \\
Total & & 40 & 60 & 100 \\
\hline
\end{tabular}

\begin{tabular}{|c|c|c|c|c|c|}
\hline \multicolumn{6}{|l|}{ Chi-Square tests } \\
\hline & Value & df & $\begin{array}{l}\text { Asymp. } \\
\text { Sig. } \\
\text { (2-sided) }\end{array}$ & $\begin{array}{l}\text { Exact } \\
\text { Sig. } \\
\text { (2-sided) }\end{array}$ & $\begin{array}{l}\text { Exact Sig. } \\
\text { (I-sided) }\end{array}$ \\
\hline $\begin{array}{l}\text { Pearson Chi- } \\
\text { Square }\end{array}$ & $.433^{\mathrm{a}}$ & 1 & 0.511 & & \\
\hline $\begin{array}{l}\text { Continuity } \\
\text { Correction }^{\mathrm{b}}\end{array}$ & 0.205 & 1 & 0.651 & & \\
\hline Likelihood Ratio & 0.434 & I & 0.51 & & \\
\hline Fisher's Exact Test & & & & 0.543 & 0.326 \\
\hline $\begin{array}{l}\text { Linear-by-Linear } \\
\text { Association }\end{array}$ & 0.429 & 1 & 0.513 & & \\
\hline $\mathrm{N}$ of Valid Cases & 100 & & & & \\
\hline
\end{tabular}

a. 0 cells $(0.0 \%)$ have expected count less than 5 . The minimum expected count is 17.60 .

b. b. Computed only for a $2 \times 2$ table.

According to the chi square test the $\mathrm{p}$ value $=0.543$ and when the $\mathrm{p}$ value is higher than 0.05 it's considered not significant. Therefore we accept the $\mathrm{H} 0$ and we reject the Ha. So we can conclude that stress does not cause loss of self-control.

\section{Discussion}

After analyzing our results using SPSS we found out that stress is caused by lacking time to exercise, lack of socializing and sleeping disturbance while overworking and un-motivation are not correlated to stress. Regarding stress outcomes we found out that overeating and loss of self-control are not associated with stress. Also we found out the most people prefer listening to music when they are stressed, and that if stress is caused by work-overload the solution is to decrease working hours.

\section{Acknowledgements}

None.

\section{Conflict of interest}

Author declares that there is no conflict of interest.

\section{References}

1. Cohen S, Janicki Deverts D, Miller GE. Psychological stress and disease. Journal of the American Medical Association. 2007;298(14):1685-1687.

2. Nordqvist C. Why stress happens and how to manage it. Medical News Today. 2017.

3. Nabi H, Kivimäki M, Batty GD, et al. Increased risk of coronary heart disease among individuals reporting adverse impact of stress on their health: the Whitehall II prospective cohort study. Eur Heart J. 2013;34(34):2697-2705.

4. Maruyama S, Morimoto K. Effects of long work hours on life-style, stress and quality of life among intermediate Japanese managers. Scand J Work Environ Health. 1996;22(5):353-359.

5. Artazcoz L, Cortès I, Borrell C, et al. Gender perspective in the analysis of the relationship between long work hours, health and health-related behavior. Scand J Work Environ Health. 2007;33(5):344-50.

6. Kim B, Joo N. Structural relations in the effect of convenience food satisfaction and quality of life according to dietary style: Comparative study of singles in metropolitan area of Korea, Japan and China. Nutr Res Pract. 2014;8(3):312-318. 